\title{
Time-Dependent Drug Administration in Hypertension and its Effect on Blood Pressure Variability
}

\author{
Annamária Magdás', Cristian Podoleanu1, Anna-Boróka Tusa², Adina Găburoi², Alexandru Incze² \\ 1 Department of Internal Medicine IV, University of Medicine and Pharmacy, Tîrgu Mureș, Romania \\ 2 Department of Internal Medicine IV, County Emergency Clinical Hospital, Tîrgu Mureș, Romania
}

\section{CORRESPONDENCE \\ Cristian Podoleanu \\ Str. Gheorghe Marinescu nr. 38 \\ 540099 Tîrgu Mureș, Romania \\ Tel: +40 744573784 \\ E-mail: podoleanu@me.com}

\section{ARTICLE HISTORY}

Received: 24 April, 2017

Accepted: 25 May, 2017
Annamária Magdás • Str. Gheorghe Marinescu nr. 38 540099 Tîrgu Mureș, Romania. Tel: +40 265215551 . E-mail: annamaria.magdas@umftgm.ro

Anna-Boróka Tusa • Str. Gheorghe Marinescu nr. 1, 540099 Tîrgu Mureș, Romania. Tel: +40 265215133 E-mail: tusa.annaa@gmail.com

Adina Găburoi • Str. Gheorghe Marinescu nr. 1, 540099 Tîrgu Mureș, Romania. Tel: +40 265215133. E-mail: adina_gaburoi@yahoo.com

Alexandru Incze • Str. Gheorghe Marinescu nr. 1 540099 Tîrgu Mureș, Romania. Tel: +40 265215133 . E-mail: alexandru.incze@umftgm.ro

\begin{abstract}
Background: Optimizing blood pressure variability seems to represent a new therapeutic target in the management of hypertension. It is emphasized that scheduling at least one antihypertensive agent at bedtime, has the ability to reduce blood pressure. Aim of the study: We have proposed to test whether the divided treatment regimen also lowers blood pressure variability. Material and methods: A number of 37 treated hypertensive patients were enrolled, who underwent 24-hour ambulatory blood pressure monitoring (ABPM) at inclusion and at 8 weeks after therapeutic intervention with divided treatment regimen. BPV was assessed using average real variability. Results: The 24-hour systolic blood presure decreased from $139.5 \pm 8.8 \mathrm{mmHg}$ to $127.4 \pm 8.9 \mathrm{mmHg}$ ( $p<0.0001$ ), and we noted a $2.1 \mathrm{mmHg}$ drop in blood pressure variability $(p<0.0001)$, as well as a two-fold increase in the percentage of dippers. After baseline ABPM assessment, in 15 cases an angiotensin-converting-enzyme-inhibitor was scheduled at bedtime, and in 10 cases it was given twice a day. In 17 cases a thiazide diuretic was administered in the afternoon, and in 18 cases a calcium channel blocker was administered at bedtime. Conclusions: For a more accurate management of patients with hypertension, the assessment of circadian blood pressure profile and the implementation of an individualized treatment that uses divided antihypertensive agents at bedtime or midday administration have the ability to optimize blood pressure control, restore dipper-profile, and decrease blood pressure variability.
\end{abstract}

Keywords: blood pressure variability, blood pressure monitoring, hypertension, average real variability

\section{INTRODUCTION}

Blood pressure (BP) undergoes significant variations over 24 hours, with a drop in the night and an increase in the early morning hours. It has been proven that patients in whom nighttime BP values do not decrease display greater cardiovascular $(\mathrm{CV})$ risk compared to dippers. ${ }^{1,2}$ Until now, in order to increase patient compliance to antihypertensive medication, a single morning dose has been applied. The pitfall of this type of dosing was that most antihypertensive agents do not have the ability to cover the entire 24-hour period. To obtain the target BP 
values in short time, the current guidelines on hypertension recommend the initiation of a combination therapy. ${ }^{3}$ The latest studies highlight the advantages of divided treatment regimen, which has the ability to cover the entire circadian BP profile. ${ }^{4,5}$

It has also been emphasized that the reduction of blood pressure variability (BPV) could be as important as BP lowering, and a potential benefit could be added by the correct timing of antihypertensive medication. ${ }^{6}$ So far, there are no randomized studies available that provide information regarding the BPV-lowering effect of time-dependent administration of medications. Therefore, the aim of this study was to analyze whether divided medication doses have the ability to reduce blood pressure variability.

\section{MATERIAL AND METHODS}

Thirty-seven treated hypertensive patients were included in this prospective study, admitted for adjustment of antihypertensive medication. The study was carried out in accordance with the World Medical Association's Declaration of Helsinki. All patients gave written informed consent, and the study protocol was approved by the ethics committee of the institution where the study was conducted.

\section{Inclusion and exclusion criteria}

We included patients with essential hypertension, according to the 2013 European Society of Hypertension/ European Society of Cardiology guidelines for the management of arterial hypertension. ${ }^{3}$ A positive diagnosis of hypertension, based on a 24-hour monitoring, required the following: awake BP $>135 / 85 \mathrm{mmHg}$ or asleep BP $>120 / 70 \mathrm{mmHg}$ or values greater than $130 / 80 \mathrm{mmHg}$ for the 24-hour period. Patients with evidence of secondary hypertension, type 1 diabetes mellitus, malignant arrhythmias, as well as shift-workers and pregnant women were excluded.

At the inclusion in the study, demographic, anthropometric, and ABPM-derived parameters, antihypertensive medication regimen, laboratory data regarding cardiovascular risk factors such as total serum cholesterol, triglycerides, fasting glucose level, and glomerular filtration rate assessed by the formula of the Modification of Diet in Renal Disease (MDRD) study were collected in a questionnaire. ${ }^{7}$

\section{4-hour ambulatory blood pressure monitoring}

Circadian blood pressure profile was assessed by 24-hour ambulatory blood pressure monitoring (ABPM), using a validated device (ABPM-05, Meditech Ltd, Hungary), at the inclusion in the study, as well as at one month after modifying the time of administration of at least one medication. The cuff size was adjusted to the patients' arm, and was fitted on the non-dominant arm. Measurements were taken every 20 minutes during daytime (06:0021:59) and nighttime (22:00-05:59). The ABPM records were accepted only if at least $80 \%$ of the scheduled measurements succeeded. Based on BP values obtained by ABPM, blood pressure variability was calculated using the formula of average real variability (ARV) presented below, where $N$ represents the number of BP measurements of the patient and $\mathrm{BP}_{k}$ is the blood pressure at measurement number $k .^{8}$

$$
A R V=\frac{1}{N-1} \sum_{k=1}^{N-1}\left|B P_{k+1}-B P_{k}\right|
$$

The 24-hour BP monitoring was repeated 8 weeks after dividing the doses into morning, midday, and bedtime administration. We did not focus on the efficacy of a given antihypertensive medication; the modification of therapy was based on the circadian BP profile of each patient.

Data were collected as raw data, and statistical analysis was performed using GraphPad Prism 5 (GraphPad Software, San Diego, USA). Numerical data were compared using the paired t-test, and categorical data were compared using the Chi-square test. The confidence interval was set at $95 \%$, and measurement data are represented as mean \pm SD.

\section{RESULTS}

A total number of 37 patients were included in the study (15 male and 22 female), with a mean age of $57.16 \pm 10.9$ years. Regarding cardiovascular risk factors assessed at inclusion, body mass index (BMI) was $30.84 \pm 3.7 \mathrm{~kg} / \mathrm{m}^{2}$, fasting glucose $111.5 \pm 46 \mathrm{mg} / \mathrm{dL}$, total serum cholesterol $205.4 \pm 46.5 \mathrm{mg} / \mathrm{dL}$, triglycerides $173.9 \pm 73.8 \mathrm{mg} / \mathrm{dL}$, glomerular filtration rate $83.9 \pm 13.8 \mathrm{~mL} / \mathrm{min} / 1.73 \mathrm{~m}^{2}$. Baseline ABPM data as well as those obtained at 8 weeks are presented in Table 1.

After modification of the treatment regimen, blood pressure variability showed a significant decrease after 8 weeks (Figure 1). The 24-hour systolic BP decreased from $139.5 \pm 8.8 \mathrm{mmHg}$ to $127.4 \pm 8.9 \mathrm{mmHg}(\mathrm{p}<0.0001)$, and we noted a $2.1 \mathrm{mmHg}$ drop in BPV ( $\mathrm{p}<0.0001)$, as well as a two-fold increase in the percentage of dippers. 
TABLE 1. ABPM data of the study group at inclusion and at 8 weeks

\begin{tabular}{lccc}
\hline & Baseline & 8 weeks control & p value \\
\hline 24-hour systolic BP, mmHg & $139.5 \pm 8.8$ & $127.4 \pm 8.9$ & $<0.0001$ \\
24- hour diastolic BP, mmHg & $80.1 \pm 10.7$ & $73.5 \pm 9.7$ & $<0.0001$ \\
Daytime systolic BP, mmHg & $143.4 \pm 10.3$ & $131.3 \pm 9.5$ & $<0.0001$ \\
Daytime diastolic BP, mmHg & $84.4 \pm 11.9$ & $76.9 \pm 11$ & $<0.0001$ \\
Nighttime systolic BP, mmHg & $133.1 \pm 9.5$ & $120.3 \pm 11.6$ & $<0.0001$ \\
Nighttime diastolic BP, mmHg & $72.6 \pm 10.3$ & $68.1 \pm 10.2$ & 0.0003 \\
Morning surge, mmHg & $21.8 \pm 12.5$ & $16.5 \pm 11.7$ & 0.07 \\
Pulse pressure, mmHg & $59.3 \pm 10.2$ & $56.5 \pm 12.9$ & 0.006 \\
Non-dippers, \% & 64.86 & 32.44 & $<0.0001$ \\
Dippers, \% & 35.14 & 67.56 & $<0.0001$ \\
\hline
\end{tabular}

At the inclusion in the study, 18 patients were taking at least three antihypertensive drugs in the morning. Based on baseline ABPM data, an angiotensin-converting-enzyme-inhibitor (ACEI) was scheduled at bedtime in 15 cases, and twice a day in 10 cases. In 18 cases, a calcium channel blocker was prescribed at afternoon or bedtime administration. The third most often used medication was a thiazide diuretic, its administration being scheduled for afternoon in 17 cases.

\section{DISCUSSION}

In this paper, we assessed the effect of divided treatment regimen on blood pressure variability using the formula of average real variability. We found that based on the patients' 24-hour BP profile, implementing divided doses of antihypertensive medication decreased not only BP values, but also BP variability. The most often used treatment regimen was an ACEI twice a day, a thiazide diuretic at midday and a calcium-channel blocker in the evening. It is emphasized that drugs are cleared more slowly during the

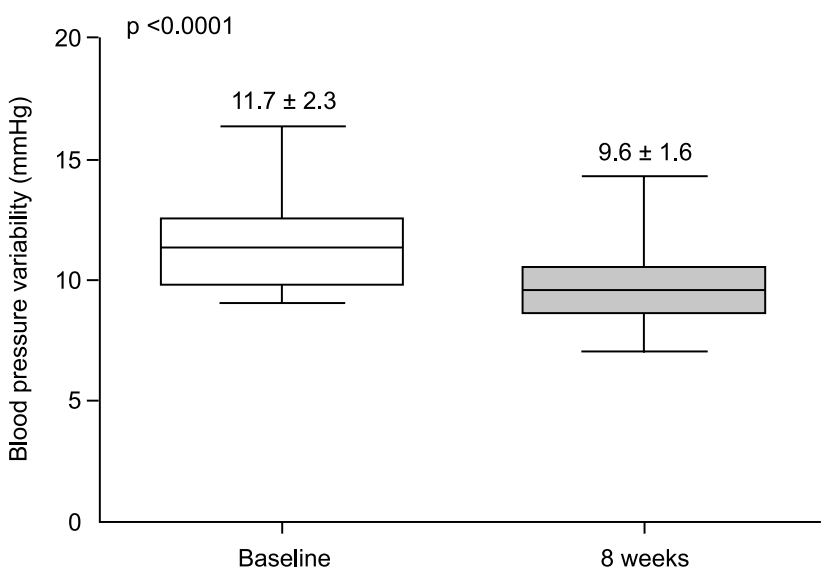

FIGURE 1. Blood pressure variability, paired t-test night, therefore it is not surprising that the ingestion of at least one antihypertensive agent at bedtime has the ability to restore the dipper pattern and decrease BPV as well as the morning surge. ${ }^{4}$

As it is seen in daily clinical practice, most antihypertensive agents that are designed for one daily administration, do not have the ability to cover the 24-hour profile. Increasing the dose or adding another short-acting agent could result in a non-dipper profile and lead to a rise in BPV. ${ }^{6}$ Therefore, the pioneer MAPEC study demonstrated that bedtime administration of medication has the ability to restore the dipper profile and prevent major cardiovascular events in the early morning period. ${ }^{5}$ To the best of our knowledge, this is the first paper that highlights the positive effect of chronotherapy on BPV. So far, average real variability as a marker of BPV has not been used to quantify the degree of BPV. A study performed by Hoshino et al, which included 31 patients, showed that by implementing chronotherapy for olmesartan and amlodipine, BPV defined as morning surge has decreased and the number of dippers has increased. ${ }^{9}$

In our study, we found a $12.1 \mathrm{mmHg}$ fall in the 24-hour systolic BP and a two-fold increase in the percentage of dippers, while BPV showed a decrease of $2.1 \mathrm{mmHg}$. These results may seem irrelevant, but as we mentioned, the randomized subjects were on treatment, and in clinical practice this value could be able to classify a patient in the high or intermediate variability group. ${ }^{10}$ We decided to use the ARV because it seems to be an accurate parameter that reflects the variability between consecutive BP values. ${ }^{8}$

The pitfall of the present study is, on one hand, the small number of participants and on the other hand, the fact that we did not assess the effect of a given antihypertensive agent on BPV, the therapeutic intervention being individualized for each patient. We did not implement a washout period because of the low compliance of the patients, 
where an unrecognized rise of $\mathrm{BP}$ values could have led to cardiovascular events.

\section{CONCLUSION}

To optimize hypertension treatment, the assessment of individual circadian BP profile and BPV is of major importance. Individualized treatment strategy with divided antihypertensive regimen should be implemented by taking into account the peak effect of each medication and the BP profile of each subject. This strategy seems to be able to decrease BP values and is effective in reducing BPV and thus improving prognosis.

\section{CONFLICT OF INTEREST}

The authors declare no conflict of interest.

\section{REFERENCES}

1. Verdecchia P, Porcellati C, Schillaci G, et al. Ambulatory blood pressure. An independent predictor of prognosis in essential hypertension. Hypertens. 1994:24:793-801
2. Carter BL, Pharm D, Chrischilles EA, et al. Efficacy and Safety of Nighttime Dosing of Antihypertensives: Review of the Literature and Design of a Pragmatic Clinical Trial. J Clin Hypertens. 2014;16:115-121.

3. Mancia G, Fagard R, Narkiewicz K, et al. 2013 ESH/ESC Guidelines for the management of arterial hypertension: the Task Force for the management of arterial hypertension of the European Society of Hypertension $(\mathrm{ESH})$ and of the European Society of Cardiology (ESC). J Hypertens. 2013:31:1281-1357.

4. Huangfu W, Duan P, Xiang D, et al. Administration time-dependent effects of combination therapy on ambulatory blood pressure in hypertensive subjects. Int J Clin Exp Med. 2015;8:19156-19161.

5. Hermida RC, Ayala DE, Mojón A, et al. Influence of circadian time of hypertension treatment on cardiovascular risk: Results of the MAPEC study. Chronobiol Int. 2010;27:1629-1651.

6. Choi HJ. Blood pressure variability and its management in hypertensive patients. Korean J Fam Med. 2012;33:330-335

7. Levey AS, Stevens LA, Schmid CH, et al. A new equation to estimate glomerular filtration rate. Ann Intern Med. 2009;150:604-612.

8. Mena LJ, Maestre GE, Hansen TW, et al. How many measurements are needed to estimate blood pressure variability without loss of prognostic information? Am J Hypertens. 2017;27:46-55.

9. Hoshino A, Nakamura T, Matsubara H. The bedtime administration ameliorates blood pressure variability and reduces urinary albumin excretion in amlodipine-olmesartan combination therapy. Clin Exp Hypertens. 2010;32:416-422.

10. Magdás A, Szilágyi L, Incze A. Can Ambulatory Blood Pressure Variability Contribute to Individual Cardiovascular Risk Stratification? Comput Math Methods Med. 2016; Article ID 7816830. 